\title{
TBCRC 018: phase II study of iniparib in combination with irinotecan to treat progressive triple negative breast cancer brain metastases
}

\author{
Carey Anders - Allison M. Deal • Vandana Abramson - Minetta C. Liu • \\ Anna M. Storniolo • John T. Carpenter - Shannon Puhalla • Rita Nanda • \\ Amal Melhem-Bertrandt • Nancy U. Lin • P. Kelly Marcom • Catherine Van Poznak • \\ Vered Stearns • Michelle Melisko $\cdot$ J. Keith Smith • Olga Karginova • \\ Joel Parker · Jonathan Berg • Eric P. Winer • Amy Peterman • Aleix Prat • \\ Charles M. Perou • Antonio C. Wolff • Lisa A. Carey
}

Received: 16 June 2014/ Accepted: 18 June 2014/Published online: 8 July 2014

(C) The Author(s) 2014. This article is published with open access at Springerlink.com

\begin{abstract}
Nearly half of patients with advanced triple negative breast cancer (TNBC) develop brain metastases (BM) and most will also have uncontrolled extracranial disease. This study evaluated the safety and efficacy of iniparib, a small molecule anti-cancer agent that alters reactive oxygen species tumor metabolism and penetrates the blood brain barrier, with the topoisomerase I inhibitor irinotecan in patients with TNBC-BM. Eligible patients had TNBC with new or progressive BM and received irinotecan and iniparib every 3 weeks. Time to progression (TTP) was the primary end point; secondary endpoints
\end{abstract}

On behalf of the Translational Breast Cancer Research Consortium.

Electronic supplementary material The online version of this article (doi:10.1007/s10549-014-3039-y) contains supplementary material, which is available to authorized users.

C. Anders - A. M. Deal · J. K. Smith · O. Karginova - J. Parker .

J. Berg · C. M. Perou · L. A. Carey

Lineberger Comprehensive Cancer Center, University of North Carolina, 450 West Drive, Campus Box 7295, Chapel Hill, NC 27599, USA

C. Anders $(\bowtie) \cdot$ L. A. Carey

Division of Hematology-Oncology, Lineberger Comprehensive Cancer Center, University of North Carolina at Chapel Hill,

170 Manning Drive, Campus Box 7305, Chapel Hill

NC 27517, USA

e-mail: carey_anders@med.unc.edu

\section{Abramson}

Vanderbilt-Ingram Cancer Center, 691 Preston Building,

Nashville, TN 37232, USA

M. C. Liu

Lombardi Comprehensive Cancer Center, Georgetown

University, 3800 Reservoir Rd, Washington, DC 20057, USA were response rate (RR), clinical benefit rate (CBR), overall survival (OS), toxicity, and health-related quality of life. Correlative endpoints included molecular subtyping and gene expression studies on pre-treatment archival tissues, and determination of germline BRCAl/2 status. Thirty-seven patients began treatment; 34 were evaluable for efficacy. Five of 24 patients were known to carry a $B R C A$ germline mutation (4 BRCA1, 1 BRCA2). Median TTP was 2.14 months and median OS was 7.8 months. Intracranial RR was $12 \%$, while intracranial $\mathrm{CBR}$ was $27 \%$. Treatment was well-tolerated; the most common grade $3 / 4$ adverse events were neutropenia and fatigue. Grade 3/4 diarrhea was rare $(3 \%)$. Intrinsic subtyping revealed 19 of 21 tumors (79\%) were basal-like, and intracranial response was associated with high expression of proliferation-related genes. This study suggests a modest

A. M. Storniolo

Indiana University Simon Cancer Center, 535 Barnhill Drive, Indianapolis, IN 46202, USA

J. T. Carpenter

University of Alabama at Birmingham, 1720 2nd Avenue South, 2508, Birmingham, AL 35294, USA

S. Puhalla

University of Pittsburgh Cancer Institute, 5150 Centre Avenue, Pittsburgh, PA 15232, USA

R. Nanda

University of Chicago, 5841 S Maryland Ave., Chicago,

IL 60637, USA

A. Melhem-Bertrandt

MD Anderson Cancer Center, 1515 Holcombe Blvd, Houston,

TX 77030, USA 
benefit of irinotecan plus iniparib in progressive TNBC-BM. More importantly, this trial design is feasible and lays the foundation for additional studies for this treatment-refractory disease.

Keywords Irinotecan - Iniparib - Brain metastases . Breast cancer $\cdot$ Phase II $\cdot$ Triple negative

$\begin{array}{ll}\text { Abbreviations } \\ \text { TNBC } & \text { Triple negative breast cancer } \\ \text { BM } & \text { Brain metastases } \\ \text { TTP } & \text { Time to progression } \\ \text { RR } & \text { Response rate } \\ \text { CBR } & \text { Clinical benefit rate } \\ \text { OS } & \text { Overall survival } \\ \text { PARP } & \text { Poly-ADP-ribose polymerase } \\ \text { TBCRC } & \text { Translational Breast Cancer Research } \\ & \text { Consortium } \\ \text { IHC } & \text { Immunohistochemistry } \\ \text { FISH } & \text { Fluorescence in situ hybridization } \\ \text { MRI } & \text { Magnetic resonance imaging } \\ \text { CSF } & \text { Cerebrospinal fluid } \\ \text { RECIST } & \text { Response Evaluation Criteria in Solid Tumors } \\ \text { CNS } & \text { Central nervous system } \\ \text { CR } & \text { Complete response } \\ \text { PR } & \text { Partial response } \\ \text { LD } & \text { Longest diameter } \\ \text { PD } & \text { Progressive disease } \\ \text { SD } & \text { Stable disease }\end{array}$

N. U. Lin · E. P. Winer

Dana-Farber Cancer Institute, 450 Brookline Ave., Boston, MA 02215, USA

P. Kelly Marcom

Duke University, 10 Bryan Searle Drive, Durham, NC 27710, USA

C. Van Poznak

University of Michigan, 1500 East Medical Center Drive, Ann Arbor, MI 48109, USA

V. Stearns · A. C. Wolff

Johns Hopkins Sidney Kimmel Comprehensive Cancer Center, 401 N Broadway, Baltimore, MD 21231, USA

M. Melisko

University of California San Francisco, Box 1710, UCSF, San Francisco, CA 94143, USA

\section{A. Peterman}

University of North Carolina at Charlotte, 9201 University City Blvd, Charlotte, NC 28223, USA

\section{A. Prat}

Vall d'Hebron Institute of Oncology, Passeig Vall d'Hebron 119, Edifici Maternoinfantil Planta 14, 08035 Barcelona, Spain

$\begin{array}{ll}\text { SAT } & \text { Segmentation Analysis and Tracking } \\ \text { NCI } & \text { National Cancer Institute } \\ \text { CTCAE } & \text { Common Toxicity Criteria for Adverse Events } \\ \text { GATK } & \text { Genome Analysis Toolkit } \\ \text { FFPE } & \text { Formalin-fixed, paraffin-embedded } \\ \text { H/E } & \text { Hematoxylin and eosin } \\ \text { ROR } & \text { Risk of relapse } \\ \text { VEGF } & \text { Vascular endothelial growth factor } \\ \text { HRQL } & \text { Health-related quality of life } \\ \text { FACT-G } & \text { Functional Assessment of Cancer Therapy - } \\ & \text { General } \\ \text { PWB } & \text { Physical well-being } \\ \text { SFWB } & \text { Social/family well-being } \\ \text { EWB } & \text { Emotional well-being } \\ \text { FWB } & \text { Functional well-being } \\ \text { SAM } & \text { Significance Analysis of Microarray } \\ \text { RPA } & \text { Recursive partitioning analysis } \\ \text { ROR } & \text { Risk of relapse } \\ \text { RANO } & \text { Response assessment in neuro-oncology } \\ \text { UNC } & \text { University of North Carolina }\end{array}$

\section{Introduction}

Brain metastases (BM) are diagnosed in approximately half of all women with advanced triple negative breast cancer (TNBC) and portend a poor prognosis [1]. Retrospective series illustrate overall survival following a diagnosis of TNBC-BM is $<4$ months despite initial treatment with whole brain radiotherapy (WBRT) [2]. Moreover, most patients $(\sim 80 \%)$ diagnosed with TNBC-BM experience new/progressive extracranial metastases such that systemic therapies capable of controlling both intra- and extracranial disease are needed [1]. In contrast to patients with endocrinesensitive or HER2-positive BC-BM, the addition of systemic therapy following WBRT has yet to yield improvements in survival for patients with TNBC-BM [3]. The development of effective systemic therapies for patients diagnosed with TNBC-BM is an urgent and unmet medical need.

Iniparib, (BSI-201, Sanofi) a pro-drug formerly thought to be an inhibitor of PARP (Poly-ADP-ribose polymerase), is currently considered to act by altering reactive oxygen species metabolism in tumor cells [4]. The physical properties of iniparib (i.e. low molecular weight [292 daltons], lipophilic nature) may facilitate blood brain barrier (BBB) penetration making it an attractive candidate to target $\mathrm{BM}$ [5]. A randomized, open-label, phase III study in metastatic TNBC of iniparib plus gemcitabine/carboplatin did not meet its progression-free (PFS) and overall survival (OS) endpoints [6]. However, a pre-specified subgroup analysis of patients treated in 2nd and 3rd-line settings, a time when 
BM often are identified, suggested an improvement in outcome by the addition of iniparib to chemotherapy.

Irinotecan is a topoisomerase I inhibitor that has activity in metastatic breast cancer with response rates of 20-30\% as a single agent and in combination with platinum-based therapy [7, 8]. Irinotecan, known to cross the BBB as evidenced by activity in primary brain tumors, has shown preclinical synergistic potential with iniparib in xenograft models of human cancer $[9,10]$. Moreover, tolerability and activity of irinotecan and iniparib have been demonstrated in the treatment of patients with extracranial metastatic breast cancer in the phase Ib setting [11].

The current multi-center, phase II study, Translational Breast Cancer Research Consortium (TBCRC) 018, was conducted to evaluate the activity and safety of iniparib and irinotecan in patients with new or progressive TNBC-BM. Recognizing that the majority of patients with TNBC-BM are concurrently diagnosed with systemic disease, the primary objective was assessment of time to progression (TTP, intracranial or extracranial). In addition to efficacy and safety analyses, we report volumetric assessment of intracranial response, patterns of disease progression, correlative endpoints from archival tumor specimens, frequency of germline $B R C A$ mutations, and quality of life among patients with TNBC-BM treated with irinotecan and iniparib.

\section{Materials and methods}

\section{Patients}

Patients were enrolled from 12 institutions from 7/2010-8/ 2012 and assigned to one of 2 cohorts: Cohort 1 those with new and/or unequivocal progressive BM treated with prior cranial radiation (i.e. WBRT and/or stereotactic radiosurgery, SRS) and Cohort 2 those with new radiotherapy-naïve $\mathrm{BM}$ for whom cranial radiation was not emergently indicated. The decision to enroll patients to Cohort 2 was made in concert with a radiation oncologist, neurosurgeon, or both.

Eligible patients had histologically-confirmed, estrogen and progesterone receptor negative $(<10 \%)$ and HER2 negative $(0-1+$ by immunohistochemistry or non-amplified by fluorescence in situ hybridization) adenocarcinoma of the breast with $\mathrm{BM}$ measuring $>5 \mathrm{~mm}$ in longest dimension on gadolinium-enhanced brain magnetic resonance imaging [MRI]. There was no limit to number of prior systemic therapies. Stable or decreasing dose of steroids for $\geq 7$ days was required. Additional inclusion criteria included age $>21$ years, ECOG performance status of $0-2$, and life expectancy of $\geq 12$ weeks. Patients were also required to have adequate organ function, and no serious infection or comorbid illness.

Exclusion criteria included pregnancy/breast-feeding, previous allergic reaction to iniparib or irinotecan, intracranial hemorrhage, impending herniation or diffuse leptomeningeal disease. Use of strong CYP3A4 inhibitors (except systemic glucocorticoids) was prohibited. All patients provided written informed consent and the study was approved by the institutional review board at each site (No. NCT01173497).

\section{Study design}

This was an open-label, single arm phase II study. The primary endpoint was TTP, extracranial or intracranial, whichever occurred first. Secondary endpoints included safety and tolerability, intracranial and extracranial response rates, PFS, OS, quality of life and genetic and genomic tissue and blood-based correlative endpoints.

Administration of study treatment

Eligible patients received irinotecan $125 \mathrm{mg} / \mathrm{m}^{2}$ intravenously (IV) days 1 and 8 of each 21 day cycle. When the study opened, iniparib was dosed at $5.6 \mathrm{mg} / \mathrm{kg}$ IV days 1,4 , 8, 11 of each 21 day cycle. In April 2011, based on emerging data in primary brain tumors, the dose of iniparib was raised to $8 \mathrm{mg} / \mathrm{kg}$ on the same schedule [12]. Patients receiving $5.6 \mathrm{mg} / \mathrm{kg}$ dosing of iniparib at that time were given the choice to dose-escalate. Dose delays of up to 3 weeks and two dose reductions of irinotecan $\left(100\right.$ and $\left.75 \mathrm{mg} / \mathrm{m}^{2}\right)$ were allowed. Dose reductions of iniparib were not allowed.

\section{Efficacy assessments}

Brain MRI were obtained every 9 weeks. Intracranial response was evaluated using modified response evaluation criteria in solid tumors (RECIST) criteria for the primary objective of TTP [13]. A central nervous system (CNS) response was defined as either a complete response $(\mathrm{CR})$ or a partial response (PR) $(>30 \%$ decrease in the sum of the longest diameter (LD) of target lesions AND an absolute decrease of $>5 \mathrm{~mm}$ in at least one target lesion). Progressive disease (PD) in the CNS was defined as $>20 \%$ increase in the sum LD of target lesions AND an absolute increase $>5 \mathrm{~mm}$ in at least one target lesion OR the appearance of one or more new lesions of at least $6 \mathrm{~mm}$ in size. Stable disease (SD) in the CNS did not meet criteria for either PR or PD.

Volumetric changes in CNS tumor burden were assessed every 9 weeks centrally at the University of North Carolina (UNC). See Supplemental Methods for detail. An intracranial response was defined as either a CR or PR, $\geq 50 \%$ (reduction in volumetric sum of all CNS lesions). PD was defined as $\geq 40 \%$ increase in the volumetric sum of all CNS lesions relative to the nadir, new CNS lesions or progression of non-measureable CNS lesions [14]. 
To assess extracranial disease, a serial computed tomography of the chest abdomen and pelvis and a nuclear bone scan (if bone metastases on baseline imaging) were obtained every 9 weeks. Extracranial disease status was assessed using RECIST 1.1 criteria [15].

\section{Safety assessments}

Adverse events were assessed every 3 weeks and graded according to the NCI Common Toxicity Criteria for Adverse Events (CTCAE), version 4.0.

Correlative studies

\section{BRCA testing}

Germline BRCAl and BRCA2 mutation testing was requested of all patients who were given options in the consent form: (1) use of known BRCA results, (2) pursue $B R C A$ counseling and testing via Myriad Genetics as per standard of care, (3) pursue BRCA testing for research purposes only, or (4) decline $B R C A$ testing.

For $n=6$ cases, BRCA testing was performed for research purposes only at UNC. DNA was extracted from whole blood using PUREGENE extraction chemistry on the Autopure LS (Qiagen, Germantown MD 20874, USA). HaloPlex Design Wizard (Agilent) was used to design 49 target regions for the BRCA1 and BRCA2 genes covering $99.4 \%(28561 \mathrm{bp})$ of the total region size. Target regions were enriched using HaloPlex Target Enrichment technology (Agilent, Santa Clara, CA, USA). Indexes were incorporated for each sample during enrichment, allowing samples to be multiplexed prior to sequencing. For full details on data processing, see Supplemental Methods.

\section{Gene expression}

Archival, formalin-fixed, paraffin-embedded (FFPE) tumor tissues were obtained and RNA was extracted (ROCHE High Pure FFPE kit, Indianapolis, IN, USA). For tissue processing, see Supplemental Methods. Expression profiling of 123 genes related to breast cancer was performed using the nCounter platform (Nanostring Technologies ${ }^{\circledR} /$ www.nanostring.com; Seattle, USA) [16], see Supplemental Data 1. Raw data was log base 2 transformed and normalized using 5 house-keeping transcripts. Gene expression and clinical data are deposited in the Gene Expression Omnibus (GSE51280).

The research-based PAM50 intrinsic subtype and claudin-low predictors were used to assign intrinsic subtype to samples [17, 18]. To account for platform-to-platform bias, a total of 53 primary breast tumors representing all intrinsic subtypes were profiled to estimate a correction factor.
We evaluated 7 different genomic signatures. From the PAM50 subtype predictor [17], we evaluated correlation to each intrinsic subtype centroid (Luminal A and B, HER2enriched, Basal-like), risk of relapse (ROR) score based on subtype and proliferation (ROR-P) and the proliferation score (mean expression of 11-proliferation related genes of the PAM50 predictor) [19]. Finally, we evaluated the VEGF-13 (vascular endothelial growth factor) gene signature [20].

Health-related quality of life

Participants' health-related quality of life (HRQL) was assessed using the Functional Assessment of Cancer Therapy, General (FACT-G) along with the Brain Tumor and Breast Cancer Additional Concerns Subscales (www. facit.org) [21-23]. See Supplemental Methods for detail. HRQL questionnaires were administered during the prestudy evaluation, every 9 weeks during treatment, at the time of progression, and at 60-day follow-up.

Statistical analysis

A sample size of 32 was chosen to achieve $81 \%$ power to detect a difference between the null hypothesis mean TTP of 2 months and alternative of 3.15 months at a 0.05 significance level (alpha) using a one-sided test based on the number of events [24]. The target enrollment was planned at 40 to account for anticipated $20 \%$ drop-out rate to result in 32 evaluable patients. The Kaplan-Meier method was used to estimate median time to event overall and for subgroups of interest, and comparisons between these subgroups were made using the Log-rank test. Both TTP and OS were calculated from start of treatment, and patients who died on study prior to progression were censored for TTP analyses. A paired $t$ test was used to compare HRQL subscale scores between the pre-study and first follow-up assessments. Fisher's exact tests were used to compare toxicities between groups. To evaluate the association of best response as determined by modified RECIST and volumetric criteria, Cox proportional hazard models were fit treating response as a time-varying covariate. The association between the expression of each single gene, as a continuous variable, and response was evaluated using an unpaired two-class Significance Analysis of Microarray (SAM) [25]. Gene ontology biological processes enriched in each gene list were identified using DAVID online tool [26].

\section{Results}

Patient characteristics

Table 1 lists the characteristics of study patients. In total, 46 patients were consented at 12 institutions between 7/2010 and 
Table 1 Demographics and patient characteristics

\begin{tabular}{|c|c|}
\hline Patient demographics $(n=37)$ & No. \\
\hline Median age (years; range) & $47(34-80)$ \\
\hline \multicolumn{2}{|l|}{ Race } \\
\hline White & $33(89 \%)$ \\
\hline Black & $4(11 \%)$ \\
\hline \multicolumn{2}{|l|}{$B R C A$ status known, $n=24$} \\
\hline Mutated (4 BRCA1, 1 BRCA2) & $5(21 \%)$ \\
\hline \multicolumn{2}{|l|}{ Stage at breast cancer diagnosis } \\
\hline I & $8(22 \%)$ \\
\hline II & $20(56 \%)$ \\
\hline III & $6(17 \%)$ \\
\hline IV & $2(6 \%)$ \\
\hline \multicolumn{2}{|l|}{ Disease status } \\
\hline $\begin{array}{l}\text { Median Time since first diagnosis of metastatic } \\
\text { breast cancer (range) }\end{array}$ & $\begin{array}{l}1.1 \text { years } \\
(0.08-7.27 \text { years })\end{array}$ \\
\hline $\begin{array}{l}\text { Median Time since first diagnosis of brain } \\
\text { metastases (range) }\end{array}$ & $\begin{array}{l}0.65 \text { years } \\
(0.04-4.01 \text { years })\end{array}$ \\
\hline $\mathrm{CNS}$ as a site at first distant recurrence & $16(43 \%)$ \\
\hline Extracranial disease at enrollment & $26(70 \%)$ \\
\hline \multicolumn{2}{|l|}{ Prior systemic chemotherapy } \\
\hline (Neo)-adjuvant & $32(87 \%)$ \\
\hline Metastatic & $25(68 \%)$ \\
\hline Prior Metastatic lines, \# (range) & $1(0-14)$ \\
\hline Prior Iniparib & $5(14 \%)$ \\
\hline \multicolumn{2}{|l|}{ Prior cranial radiotherapy } \\
\hline Cohort 1 (prior cranial radiation) & $31(84 \%)$ \\
\hline WBRT & $13(42 \%)$ \\
\hline Radiosurgery & $7(23 \%)$ \\
\hline Both WBRT and Radiosurgery & $11(36 \%)$ \\
\hline Cohort 2 (radiation therapy naïve) & $6(16 \%)$ \\
\hline Prior neurosurgery & $10(27 \%)$ \\
\hline \multicolumn{2}{|l|}{ Recursive partitioning analysis (RPA) score } \\
\hline 1 & $7(19 \%)$ \\
\hline 2 & $22(59 \%)$ \\
\hline 3 & $8(22 \%)$ \\
\hline Baseline Abnormal Neurologic exam & $16(43 \%)$ \\
\hline Steroid use at baseline & $23(62 \%)$ \\
\hline \multicolumn{2}{|l|}{ Iniparib dose } \\
\hline $5.6 \mathrm{mg} / \mathrm{kg}$ & $23(62 \%)$ \\
\hline $8 \mathrm{mg} / \mathrm{kg}$ & $9(24 \%)$ \\
\hline Both & $5(14 \%)$ \\
\hline
\end{tabular}

8/2012. Nine patients were consent-not-treat ( $n=7$ with PD during screening, $n=1$ with Gilbert's Syndrome, $n=1$ with hemorrhage on baseline brain MRI). Thirty-seven patients were evaluable for toxicity and 34 for efficacy. Of the 24 patients for whom $B R C A$ status was known, $21 \%$ harbored a germline mutation (4 BRCA1, 1 BRCA2). Recursive partitioning analysis (RPA) score was calculated and was 1,2 and 3 in 19, 59 and $22 \%$ of patients, respectively [27].

Toxicity and dose Intensity

Irinotecan plus iniparib was generally well-tolerated $(n=37$, Fig. 1$)$. The most common grade $1 / 2$ adverse events were diarrhea (51\%), nausea (46\%), fatigue (32\%), neutropenia (22\%), and constipation ( $22 \%)$. The most common grade 3 or greater adverse events were neutropenia (14\%), leukopenia (5\%) hypokalemia (5\%), and decreased white blood cells $(5 \%)$.

Disease progression as defined by radiographic imaging was the most common reason for treatment discontinuation $(27 / 34,79 \%)$. Four patients (12\%) experienced functional decline in the absence of radiographic progression. No patient had a serious adverse event that led to study withdrawal. One patient died while on protocol due to overwhelming respiratory infection unrelated to treatment, one withdrew for personal reasons, and one remained on protocol therapy at the time of analysis.

Eight of 34 patients evaluable for efficacy (24\%) required an initial dose modification of irinotecan to $100 \mathrm{mg} / \mathrm{m}^{2}$, and 3 of 8 required a second dose modification to $75 \mathrm{mg} / \mathrm{m}^{2}$. Seventeen $(50 \%)$ required a dose delay due to hematologic toxicity. Of the five patients who escalated the dose of iniparib to $8 \mathrm{mg} / \mathrm{kg}$ mid-study, one patient deescalated back down to $5.6 \mathrm{mg} / \mathrm{kg}$ due to grade 2 nausea. There was no difference between grade $3 / 4$ adverse events by dose of iniparib $(5.6 \mathrm{mg} / \mathrm{kg}$ vs. ever receiving $8 \mathrm{mg} / \mathrm{kg}$, $p=0.27)$ or by BRCA mutation status $(p=0.99)$.

\section{Efficacy}

\section{Time to progression}

Among 34 evaluable patients, 32 (94\%) experienced either intracranial or extracranial progression (or both), one died on study, and the other remained on treatment as part of an extension study. The median TTP was 2.14 months (95\% CI 1.74-4.34, Fig. 2a). In exploratory analysis, there was a significant improvement in TTP by iniparib dose $(p=0.009)$. Median TTP was 7.8 months for 9 patients who received $8 \mathrm{mg} / \mathrm{kg}$, 5.7 months for 5 who received both doses, and 1.9 months for 23 who received $5.6 \mathrm{mg} / \mathrm{kg}$. There was no difference in TTP by $B R C A$ mutation status (2.3 months for $B R C A$ wild-type vs. 1.6 months for $B R C A$ mutation carriers, $p=0.91$ ).

\section{Overall survival}

At the time of the analysis, 27 (79\%) of patients had died. Median OS was 7.83 months (95\% CI 5.10-10.2, Fig. 2b). 
There was no difference in OS by either iniparib dosing $(p=0.24)$ or BRCA mutation status $(p=0.49)$.

\section{Objective response rate}

Of 34 evaluable patients, the intracranial response rate was $12 \%$ (0 CR, 4 PR's, Table 2). Thirteen additional patients (41\%) had SD as best response intracranially, while for 15 (47 \%), it was PD. The intracranial CBR, including those with PR and SD for $\geq 6$ months, was $27 \%$ (9/34). Of the $n=4$ patients who achieved an intracranial PR, 2 harbored a BRCAl mutation. Extracranial disease was evaluable in 19 patients, of whom 1 had a PR (5\%). Six patients (32\%) experienced extracranial SD, while $12(63 \%)$ experienced $\mathrm{PD}$ as best response. The extracranial CBR was $11 \%$ $(2 / 19)$. Site of first progression was intracranial in $38 \%$,

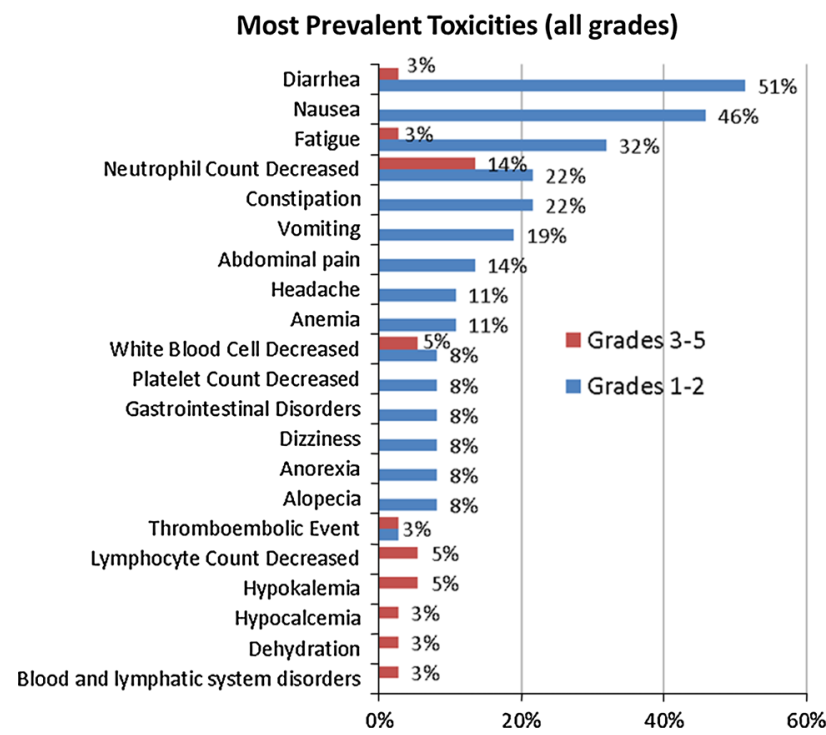

Fig. 1 Most prevalent toxicities (All grades) in response to irinotecan and iniparib therapy. Grade 1 and 2 toxicities are presented in blue; Grade 3-5 toxicities are presented in red

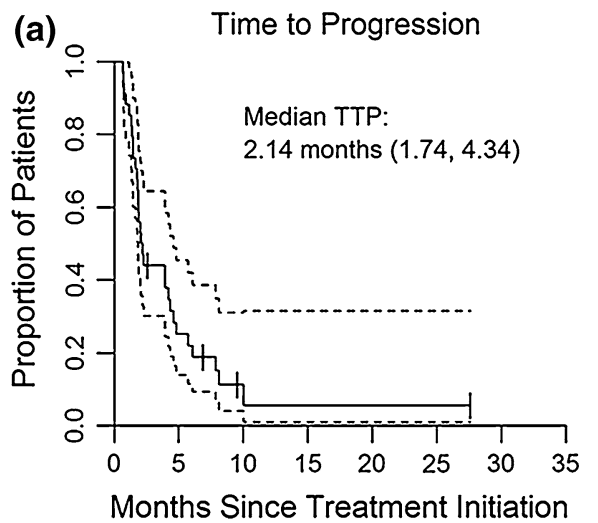

extracranial in $31 \%$ and both intra- and extracranial in $31 \%$.

\section{Volumetric analysis of intracranial response}

Intracranial response was evaluated using uni-dimensional (modified RECIST) [13] and three-dimensional measurements (volumetric criteria) [14]. Intracranial PR by volumetric analysis ( $\geq 50 \%$ reduction) was observed in 6 patients $(22 \%)$, SD in $11(41 \%)$ and $\mathrm{PD}(\geq 40 \%$ increase) in $10(37 \%)$ (Fig. 3).

Comparing intracranial response by modified RECIST and volumetric criteria, discordance in response classification was observed $(10 / 27,37 \%)$. It was more common for volumetric response to overestimate response when compared to uni-dimensinoal response classification ( $7 / 10$, $70 \%$ ), see Supplemental Table 1 and Supplemental Results. Achieving a PR by RECIST criteria was significantly associated with $\mathrm{OS} \quad(\mathrm{HR}=0.12,95 \%$ CI $0.015-0.877, p=0.037$ ), while a PR as determined by volumetric criteria did not $(\mathrm{HR}=0.41,95 \%$ CI $0.120-1.39, p=0.15)$.

Health-related quality of Life (HRQL)

Of 37 patients evaluable for toxicity, 11 completed the baseline HRQL only, and 25 completed at least a baseline and one follow-up HRQL. For details on participants, see Supplemental Results. To provide a detailed picture of subjects' HRQL, FACT subscales were all analyzed separately. For the entire population, the only significant change was in physical well-being, which declined from pre-study to first follow-up HRQL assessment $(p<0.01)$. We compared the change in HRQL from pre-study to first follow-up between subjects who received a clinical benefit from the treatment versus those who did not and found no significant difference, Supplemental Table 2.

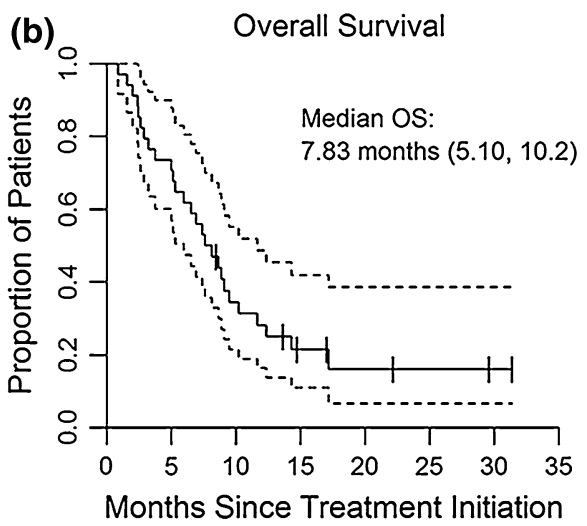

Fig. 2 a Median time to progression (TTP) and b median overall survival (OS) in response to irinotecan and iniparib among patients with progressive or new brain metastases arising from triple negative breast cancer 
Table 2 Summary of objective response rates

\begin{tabular}{lll}
\hline & $\begin{array}{l}\text { Intracranial } \\
(n=34) n^{\mathrm{a}}\end{array}$ & $\begin{array}{l}\text { Extracranial } \\
(n=19) n(\%)\end{array}$ \\
\hline Complete response & 0 & 0 \\
Partial response & $4(12 \%)$ & $1(5 \%)$ \\
Stable disease & $13(41 \%)$ & $6(32 \%)$ \\
$\begin{array}{l}\text { Progressive disease } \\
\text { Clinical benefit rate }(\mathrm{CR}\end{array}$ & $15(47 \%)$ & $12(63 \%)$ \\
or PR + SD $>6$ months $)$ & $9(27 \%)$ & $2(11 \%)$ \\
\hline
\end{tabular}

${ }^{a} n=2$ pts did not have an end of treatment brain MRI as they progressed extracranially in $<6$ months. Both were classified as intracranial "No Clinical Benefit" and extracranial PD

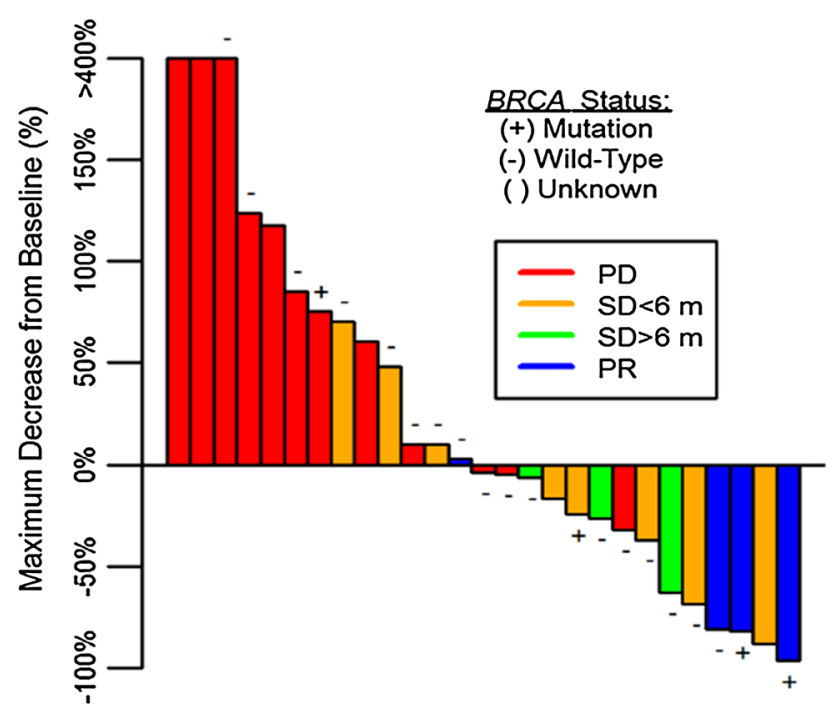

Fig. 3 Best percentage of CNS volumetric change from baseline. Response criteria defined by modified RECIST: Red progressive disease (PD), gold stable disease (SD) $<6$ months, green $\mathrm{SD}>6$ months, blue partial response (PR). BRCA mutation status indicated as follows: (+) BRCA mutation carrier, (-) wild-type BRCA, () unknown

Intrinsic subtyping and correlative endpoints

FFPE tumor was available from 21 patients, of which 24 total samples were analyzed (17 primary breast tumors, 2 lymph nodes, 2 brain metastases and 3 lung metastases). Three were matched pairs; 2 breast primary/lymph node pairs, 1 breast primary/lung metastases from the same patient.

Intrinsic molecular subtyping identified $19(79 \%)$ basal-like, 2 (8\%) HER2-enriched, and 3 (13\%) normallike tumors. Concordant with a previous molecular characterization of TNBC [28], the two HER2-enriched tumor samples did not show high ERBB2 gene expression, but showed high $E G F R$ gene expression. Notably, no luminal A, B or claudin-low tumors were identified. Subtype concordance between the 3 matched pairs was $30 \%$ with 1 breast/lymph node pair both basal-like, 1 breast/lung metastases pair basal-like/normal-like and 1 breast/lymph node pair basal-like/HER2-enriched.

We evaluated the association between expression of 7 gene signatures (as a continuous variable) and intracranial response (PR vs. SD/PD). High expression of proliferation and ROR-P scores were significantly associated ( $p=0.046$, Mann-Whitney test) with a PR (Fig. 4). When the previously defined cutoffs to define low/medium/high ROR-P expression were evaluated [19], 4/10 patients with tumors classified as ROR-P high had an intracranial PR versus 0/9 patients with tumors classified as ROR-P medium (unilateral $p=0.0576$, Fisher's exact test). No tumor was classified as RORP-low. There was no association between response and the VEGF-13 gene signature.

Secondly, we evaluated the association between expression of 123 breast cancer-associated candidate genes, and 5 house-keeping genes (as a continuous variable) and intracranial response (PR vs. SD/PD; PR vs. PD and PR vs. SD) (Supplemental Data 2). The comparison that yielded the largest number of significant genes $(n=38,30.9 \%)$ was PR vs. PD [false discovery rate (FDR) $<30 \%$ ]. Concordant with the previous findings using gene signatures, the 13 genes (e.g. NDC80 and EXO1) whose high expression was associated with an intracranial PR were found to be enriched for cell cyclerelated biological processes $(p<0.0001)$ (Supplemental Fig. 1). Conversely, the list of 25 genes whose high expression was associated with intracranial PD was found to be enriched for regulation of cytoskeleton-related (e.g. $C A V 1$ and MAPT) and transcription-related (e.g. FOXAl and $R B$ ) biological processes. Finally, the other two possible comparisons (i.e. PR vs. SD/PD and PR vs. SD) only yielded genes whose high expression was associated with lack of PR (FDR $<30 \%$ ). Interestingly, both of these gene lists were enriched for genes typically associated with HER2-enriched biology (e.g. FGFR4 and TMEM45B) and luminal biology (e.g. INPP4B, FOXAl, NATl and PGR).

\section{Discussion}

Systemic therapeutic options for patients with TNBC brain metastases (TNBC-BM) are limited, especially post-radiation therapy. In this prospective, multi-center, phase II trial of irinotecan/iniparib designed for patient with progressive TNBC-BM, intracranial CBR was $\sim 30 \%$. The pre-specified efficacy criteria was not met with a median TTP of 2.1 months (hypothesis 3.15 months); median OS was $\sim 8$ months. Survival from the current trial are consistent with prior studies of systemic therapy to treat extracranial TNBC where PFS and OS ranged from 1.5-3.1 to 10-12 months, respectively [29, 30]. Specific to BM, a recent 
Fig. 4 Correlations between intracranial response rates, $B R C A$ mutation status, intrinsic breast cancer subtype call, ROR-P (risk of recurrenceproliferation), proliferation and VEGF-13 gene signatures. High signature expression is represented by red, medium by black and low by green. Asterisk denotes a statistically significant association

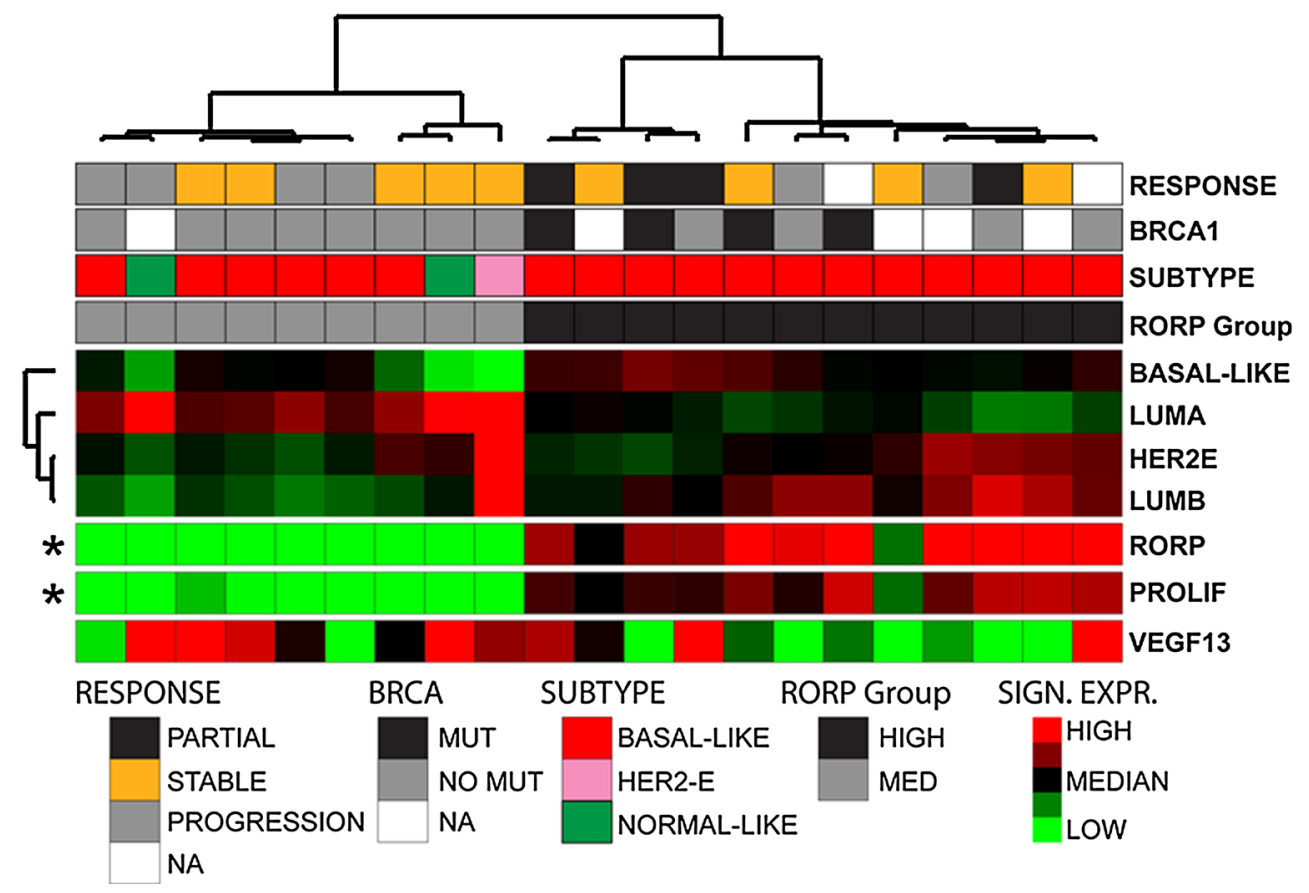

systemic therapy trial for patients with HER2-negative progressive breast cancer BM reported a PFS of 3.7 months despite a $63 \%$ intracranial RR [31]. Taken together, these results speak to the treatment-refractory nature of advanced TNBC and the dire need for improved therapies.

To our knowledge, this is the largest prospective trial conducted for patients with new and/or progressive TNBC$\mathrm{BM}$. The selection of irinotecan and iniparib was based on several criteria. First, at the time of the trial's conception, both agents had shown activity in the treatment of advanced breast cancer. Irinotecan administered on a weekly schedule yielded response rates of $>20 \%$ and iniparib had shown a $\mathrm{PFS} / \mathrm{OS}$ advantage in advanced TNBC when combined with DNA-damaging chemotherapeutics in the phase II setting [7, 32]. Second, both agents had been shown to cross the BBB making them attractive agents to combine in the setting of intra- and extracranial TNBC $[5,10]$. Third, the combination of iniparib/irinotecan was shown to be synergistic preclinically and safe in the phase I setting $[9,11]$. During the course of the study, further characterization of iniparib's mechanism of action emerged. Iniparib is now considered to act by altering reactive oxygen species metabolism in tumor cells, not through inhibition of PARP [4]. A randomized phase III trial of chemotherapy \pm iniparib in first or second line treatment of advanced TNBC did not show efficacy in first line; however, clinical benefit was suggested in later lines of treatment when BM are more commonly diagnosed [6]. As such and in the context of the current trial, the additional contribution of iniparib to irinotecan remains uncertain.
Several aspects of this trial set it apart from others. First, we observed of 4 patients with intracranial PR, $50 \%$ harbored a $B R C A$ mutation. While the sample is too small to draw definitive conclusions, there is a possible association between dysfunctional homologous recombination and sensitivity to irinotecan and/or iniparib. Secondly, gene expression analyses illustrated basal-like and/or proliferation-related gene signatures were associated with intracranial response. This is congruent with a prior report showing chemotherapy sensitivity was associated with high expression of basal-like and/or proliferation-related genes in basal-like breast cancer [33]. Finally, a 30 \% discordance between uni-dimensional and volumetric intracranial response assessment was striking, as was the positive correlation between modified RECIST response classification and OS. The most accurate manner in which to measure intracranial lesion response remains under debate [34].

To our knowledge, this is the largest series of TNBC-BM patients treated in a uniform manner, however we acknowledge limitations including small sample size and a non-randomized approach. As one of our goals was to show feasibility of enrolling this patient population to a phase II study, we selected a single-arm study design powered against historical controls such that a smaller sample size was required. This study demonstrates feasibility of successfully enrolling patients with TNBC-BM to a prospective trial.

In summary, this novel, phase II study illustrates that irinotecan and iniparib yields an intracranial CBR of $\sim 30 \%$ among patients with new and/or progressive TNBC-BM with a tolerable safety profile and no significant 
detriment to quality of life. Intracranial response was associated with the most highly proliferative tumors. Based on our findings, larger prospective studies evaluating novel agents in the setting of TNBC-BM could be coupled with irinotecan. Finally, the importance of archival tissue collection and thoughtful correlative studies in future studies cannot be over-emphasized.

Acknowledgments Ignacio Garcia-Ribas MD, Robyn Burns, Jeanne Noe PharmD, Diane Winans, Stephanie Shapiro, Rachel Phipps, Melissa Haines and Sarah Gallagher. We also thank Breast Cancer Research Foundation, Susan G. Komen for the Cure, and Avon Foundation for Women for their support of the TBCRC.

Funding NIH K23157728 (CKA): Research reported in this publication was supported by the National Cancer Institute of the National Institutes of Health under Award Number K23CA157728. The content is solely the responsibility of the authors and does not necessarily represent the official views of the National Institutes of Health. Carey K. Anders, MD of Awardee is a Damon Runyon Clinical Investigator supported (in part) by the Damon Runyon Cancer Research Foundation (CI-64-12). The clinical operations of this study were financially supported by Sanofi.

Conflict of interest CKA and LAC are uncompensated advisors/ consultants to Sanofi; CKA receives research funding from Sanofi; JTC receives an honorarium from Genomic Health; JTC and CMP receive a consultation fee from Sanofi; SP is a consultant to Pfizer, MedImmune, and Celldex; NUL receives research funding from Genentech, GlaxoSmithKline, Array Biopharma, Novartis, Geron; JP is an author on patent filings for PAM50; VS receives research funding from Abbvie, Celgene, Medimmune, Merck, Novartis and Pfizer; APrat serves in an Uncompensated advisory role for Nanostring Technologies; CMP is an equity stock holder, and Board of Directors Member of BioClassifier LLC, University Genomics, and GeneCentric Diagnostics. CMP is also listed as an inventor on a patent application on the PAM50 molecular assay and the 13-gene hypoxia signature; AMD, VA, MCL, AMS, RN, AMB, PKM, CV, MM, JKS, OK, JB, EPW, APeterman, and ACW report no competing interests.

Open Access This article is distributed under the terms of the Creative Commons Attribution Noncommercial License which permits any noncommercial use, distribution, and reproduction in any medium, provided the original author(s) and the source are credited.

\section{References}

1. Lin NU et al (2008) Sites of distant recurrence and clinical outcomes in patients with metastatic triple-negative breast cancer. Cancer 113(10):2638-2645

2. Niwińska A, Murawska M, Pogoda K (2010) Breast cancer brain metastases: differences in survival depending on biological subtype, RPA RTOG prognostic class and systemic treatment after whole-brain radiotherapy (WBRT). Ann Oncol 21(5):942-948

3. Niwińska A, Murawska M, Pogoda K (2010) Breast cancer subtypes and response to systemic treatment after whole-brain radiotherapy in patients with brain metastases. Cancer 116(18):4238-4247

4. Licht S, et al. (2011) Mechanism of action of iniparib: stimulation of reactive oxygen species (ROS) production in an iniparib-sensitive breast cancer cell line. Mol Cancer Ther 10(11 Suppl: Abstract A226)
5. Castro M, Li L, Stallings T (2010) Pharmacokinetics of BSI-201, a poly (ADP-ribose) polymerase-1 (PARP1) inhibitor, in cerebrospinal fluid (CSF) of a patient with breast cancer with carcinomatous meningitis. J Clin Oncol (Meet Abstr) 28:e13559

6. O'Shaughnessy J et al (2011) A randomized phase III study of iniparib (BSI-201) in combination with gemcitabine/carboplatin (G/C) in metastatic triple-negative breast cancer (TNBC). J Clin Oncol 29(S):1007

7. Perez EA et al (2004) Randomized phase II study of two irinotecan schedules for patients with metastatic breast cancer refractory to an anthracycline, a taxane, or both. J Clin Oncol 22(14):2849-2855

8. O'Shaughnessy J, et al. (2007) Preliminary results of a randomized phase II study of weekly irinotecan/carboplatin with or without cetuximab in patients with metastatic breast cancer. Breast Cancer Res Treat 106(Suppl 1 Abstract 308)

9. BiPar (2011) BSI-201 Investigator's Brochure

10. Vredenburgh JJ et al (2007) Bevacizumab plus irinotecan in recurrent glioblastoma multiforme. J Clin Oncol 25(30):4722-4729

11. Moulder S, et al. (2010) A phase 1b study to assess the safety and tolerability of iniparib (BSI-201) in combination with irinotecan for the treatment of metastatic breast cancer. In: San Antonio breast cancer symposium. 2010

12. Blakeley J et al (2010) Poly (ADP-ribose) polymerase-1 (PARP1) inhibitor BSI-201 in combination with temozolomide (TMZ) in malignant glioma. J Clin Oncol 28(15S):2012A

13. Lin NU et al (2008) Phase II trial of lapatinib for brain metastases in patients with human epidermal growth factor receptor 2-positive breast cancer. J Clin Oncol 26(12):1993-1999

14. Lin NU et al (2009) Multicenter phase II study of lapatinib in patients with brain metastases from HER2-positive breast cancer. Clin Cancer Res 15(4):1452-1459

15. Eisenhauer E et al (2009) New response evaluation criteria in solid tumours: revised RECIST guideline (version 1.1). Eur J Cancer 45(2):228-247

16. Geiss GK et al (2008) Direct multiplexed measurement of gene expression with color-coded probe pairs. Nat Biotechnol 26(3): 317-325

17. Parker JS et al (2009) Supervised risk predictor of breast cancer based on intrinsic subtypes. J Clin Oncol 27(8):1160-1167

18. Prat A et al (2010) Phenotypic and molecular characterization of the claudin-low intrinsic subtype of breast cancer. Breast Cancer Res 12(5):R68

19. Nielsen TO et al (2010) A comparison of PAM50 intrinsic subtyping with immunohistochemistry and clinical prognostic factors in tamoxifen-treated estrogen receptor-positive breast cancer. Clin Cancer Res 16(21):5222-5232

20. Hu Z et al (2009) A compact VEGF signature associated with distant metastases and poor outcomes. BMC Med 7(1):9

21. Brady MJ et al (1997) Reliability and validity of the functional assessment of cancer therapy-breast quality-of-life instrument. J Clin Oncol 15(3):974-986

22. Weitzner MA et al (1995) The functional assessment of cancer therapy (FACT) scale. Development of a brain subscale and revalidation of the general version (FACT-G) in patients with primary brain tumors. Cancer 75(5):1151-1161

23. Cella DF et al (1993) The Functional Assessment of Cancer Therapy scale: development and validation of the general measure. J Clin Oncol 11(3):570-579

24. Epstein B (1960) Statistical life test acceptance procedures. Technometrics 2.4(4):435-446

25. Tusher VG, Tibshirani R, Chu G (2001) Significance analysis of microarrays applied to the ionizing radiation response. Proc Natl Acad Sci 98(9):5116-5121

26. Huang DW, Sherman BT, Lempicki RA (2008) Systematic and integrative analysis of large gene lists using DAVID bioinformatics resources. Nat Protoc 4(1):44-57 
27. Gaspar LE et al (2000) Validation of the RTOG recursive partitioning analysis (RPA) classification for brain metastases. Int $\mathbf{J}$ Radiat Oncol Biol Phys 47(4):1001-1006

28. Prat A et al (2013) Molecular characterization of basal-like and non-basal-like triple-negative breast cancer. Oncologist 18(2): 123-133

29. Carey LA et al (2012) TBCRC 001: randomized phase II study of cetuximab in combination with carboplatin in stage IV triplenegative breast cancer. J Clin Oncol 30(21):2615-2623

30. Baselga J, et al. (2013) Randomized phase II study of the antiepidermal growth factor receptor monoclonal antibody cetuximab with cisplatin versus cisplatin alone in patients with metastatic triple-negative breast cancer. J Clin Oncol 31(20):2586-2592
31. Lin N, Gelman R, Younger W et al (2013) Phase II trial of carboplatin (C) and bevacizumab (BEV) in patients (pts) with breast cancer brain metastases (BCBM). J Clin Oncol 31 (suppl; abstr 513)

32. O'Shaughnessy $\mathbf{J}$ et al (2011) Iniparib plus chemotherapy in metastatic triple-negative breast cancer. N Engl J Med 364(3): 205-214

33. Prat A, et al. (2012) Gene expression-based predictors of chemotherapy response in basal-like breast cancer. J Clin Oncol 30 (suppl; abstr 10500)

34. Lin NU et al (2013) Challenges relating to solid tumour brain metastases in clinical trials, part 2: neurocognitive, neurological, and quality-of-life outcomes. A report from the RANO group. Lancet 14(10):e407-e416 